\title{
Free electron lasers with slowly varying beam and undulator parameters
}

\author{
Z. Huang and G. Stupakov \\ Stanford Linear Accelerator Center, Stanford, California 94309, USA
}

(Received 13 December 2004; published 14 April 2005)

\begin{abstract}
A self-consistent theory of a free electron laser (FEL) with slowly varying beam and undulator parameters is developed using the WKB approximation. The theory is applied to study the performance of a self-amplified spontaneous emission (SASE) FEL when the electron beam energy varies along the undulator as would be caused by vacuum pipe wakefields and/or when the undulator strength parameter is tapered in the small signal regime before FEL saturation. We find that a small energy gain or an equivalent undulator taper slightly reduces the power gain length in the exponential growth regime and can increase the saturated SASE power by about a factor of 2. Power degradation away from the optimal performance can be estimated based upon knowledge of the SASE bandwidth. The analytical results, which agree with numerical simulations, are used to optimize the undulator taper and to evaluate wakefield effects.
\end{abstract}

DOI: $10.1103 /$ PhysRevSTAB.8.040702

PACS numbers: $41.60 . \mathrm{Cr}$

\section{INTRODUCTION}

High-gain free electron lasers (FELs) are being developed as extremely bright $\mathrm{x}$-ray sources of a next-generation radiation facility. An x-ray FEL based on self-amplified spontaneous emission (SASE) typically requires an electron beam with a few kiloampere peak current and a smallgap undulator system of tens to $100 \mathrm{~m}$ in length. The collective interaction of a high-current short electron bunch with the undulator vacuum chamber may significantly change the beam energy inside the undulator and degrade the FEL performance, as highlighted by the recent analysis of the ac resistive wall wakefield [1] for the Linac Coherent Light Source (LCLS) [2]. Understanding the effects of the undulator wakefield is of critical importance in the design of an X-ray FEL.

The wakefield generates an energy variation along the undulator distance as well as along the bunch position. Since the typical bunch length for an x-ray FEL greatly exceeds the radiation slippage length over the entire undulator, the energy variation within a FEL slippage length (known as a FEL slice) is usually negligible for the wakefields that do not vary rapidly inside the bunch. Thus, the main effect of the undulator wakefield in a FEL slice is due to the energy change along the undulator distance and may be considered to be equivalent to that caused by tapering the undulator strength parameter. However, the classical treatment of a tapered undulator [3] has been focused on the FEL saturation regime where a significant energy loss induced through the FEL interaction can be offset by tapering the undulator parameter. On the other hand, the bulk of energy change due to the undulator wakefield occurs in the small signal regime before saturation, and its effect upon FEL performance has mainly been addressed by time-dependent simulation codes (see, e.g., Refs. [4,5]).

Motivated by these considerations, we present an analytical description of the FEL process in the small signal regime with slowly varying beam energy and undulator parameter. Since the rate of the wakefield-induced fractional energy change is typically less than the FEL Pierce parameter $\rho\left(\sim 10^{-3}\right.$ for short-wavelength FELs) within one electric field gain length, we develop the WKB approximation for the coupled Maxwell-Vlasov equations in order to determine the evolution of the beam-radiation system, based upon a priori knowledge of the FEL dispersion relation. In the lowest-order approximation, the growth rate $\operatorname{Im}\left(\mu_{0}\right)$ for the radiation field amplitude at a given frequency $\omega$ becomes a function of the undulator distance $z$, and the total gain is determined by $\int^{z} \operatorname{Im}\left[\mu_{0}\left(\omega, z^{\prime}\right)\right] d z^{\prime}$. The zeroth-order growth rate $\operatorname{Im}\left[\mu_{0}(\omega, z)\right]$ is obtained by shifting the growth rate of a constant-parameter FEL $\operatorname{Im}\left[\mu_{c}(\omega)\right]$ by $\Delta \omega(z)$ due to changes in the beam energy and the undulator parameter (see Fig. 1), i.e., $\operatorname{Im}\left[\mu_{0}(\omega, z)\right]=\operatorname{Im}\left[\mu_{c}(\omega-\Delta \omega(z))\right]$. In addition to the zeroth-order gain, we also find a first-order

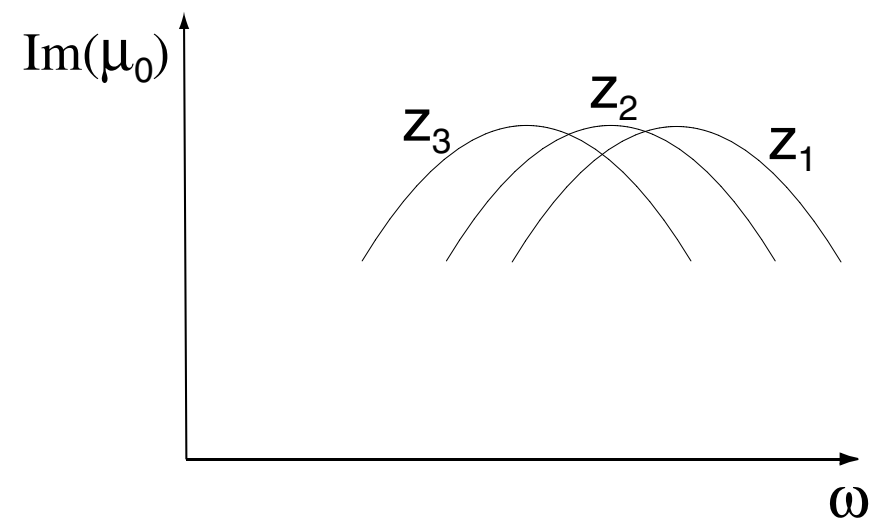

FIG. 1. Zeroth-order growth rate $\operatorname{Im}\left(\mu_{0}\right)$ as a function of the radiation frequency $\omega$ at three different undulator locations $z_{1}$, $z_{2}$, and $z_{3}$ is obtained by shifting the gain curve of a constantparameter FEL along the horizontal axis by $\Delta \omega(z)$ due to changes in the beam energy and the undulator parameter. 
correction $\mu_{1}$ that is small in comparison with $\mu_{0}$. Nevertheless, after integration over the length of the undulator, this correction can give rise to a noticeable change of the radiation power at the end of the undulator.

We apply this theory to study the SASE FEL under a linear energy variation along the undulator distance and find that a fractional energy gain of about $2 \rho$ over the saturation distance or an equivalent undulator taper can slightly reduce the gain length in the exponential growth regime and improve the saturated power by about a factor of 2 as compared to a constant-parameter FEL. Power degradation away from this optimal energy gain is approximately Gaussian with a FWHM fractional energy variation of about 4 times the relative rms radiation bandwidth, which is typically close to $\rho$ at saturation. Thus, a noticeable power degradation will occur if the accumulated fractional energy change is either negative or positive but larger than $4 \rho$.

This paper is organized in the following manner. In Sec. II, we define the problem by writing down the FEL equations with variable beam energy and undulator parameter. In Sec. III, we ignore the transverse motion of electrons and the radiation diffraction to obtain the WKB solution for the one-dimensional (1D) FEL system. We apply this solution to study the effect of a linear energy change on both seeded and SASE FELs. The results obtained in the $1 \mathrm{D}$ case are then generalized to the threedimensional (3D) system in Sec. IV and are applied to study the effects of the LCLS undulator wakefields in Sec. V. Finally, a general discussion of the WKB approximation using the matrix formalism and its application to the 3D FEL system is presented in Appendices A and B.

\section{FEL EQUATIONS WITH VARIABLE BEAM ENERGY AND UNDULATOR PARAMETER}

Let us consider a planar undulator with a period $\lambda_{u}=$ $2 \pi / k_{u}$ and an undulator strength parameter $K(z)$ that may vary along the undulator distance $z$. We also assume $\gamma_{c}(z) m c^{2}$ is the average electron energy in the absence of the FEL interaction, which may vary along the undulator due to wakefields and emission of spontaneous radiation. The initial resonant wavelength of the FEL is

$$
\lambda_{0}=\frac{2 \pi}{k_{0}}=\frac{2 \pi c}{\omega_{0}}=\frac{\lambda_{u}}{2 \gamma_{c}(0)^{2}}\left[1+\frac{K(0)^{2}}{2}\right] .
$$

We define the electron energy (in units of $m c^{2}$ ) resonant to $\lambda_{0}$ as the resonant energy:

$$
\gamma_{r}(z)=\sqrt{\frac{\lambda_{u}}{2 \lambda_{0}}\left[1+\frac{K(z)^{2}}{2}\right]},
$$

from which we obtain $\gamma_{r}(0)=\gamma_{c}(0) \equiv \gamma_{0}$.

In this and the following sections, we ignore any transverse effect and consider a 1D FEL system. The longitudinal motion of the electron with a wiggle-averaged position $c t^{*}$ can be described by a ponderomotive phase variable $\theta(z)=\left(k_{0}+k_{u}\right) z-k_{0} c t^{*}$ and a normalized energy variable $\eta(z)=\left[\gamma(z)-\gamma_{c}(z)\right] / \gamma_{0}$. Taking into account that

$$
\frac{c d t^{*}}{d z}=1+\frac{1+K(z)^{2} / 2}{2 \gamma(z)^{2}},
$$

and that changes in $K$ and $\gamma_{c}$ over the entire undulator distance are typically very small compared to $K(0) \equiv K_{0}$ and $\gamma_{0}$, the FEL pendulum equations [6] can be written as

$$
\begin{aligned}
\frac{d \theta}{d z}= & 2 k_{u} \frac{\gamma(z)-\gamma_{r}(z)}{\gamma_{0}}=2 k_{u}(\eta+\delta), \\
\frac{d \eta}{d z}= & \frac{e K_{0}[\mathrm{JJ}]}{4 \gamma_{0}^{2} m c^{2}} \int d \nu E_{\nu}(z) e^{i \nu \theta-i \Delta \nu k_{u} z} \\
& + \text { complex conjugate, }
\end{aligned}
$$

where the fractional energy change with respect to the resonant energy in the absence of the FEL interaction is

$$
\delta(z)=\frac{\gamma_{c}(z)-\gamma_{r}(z)}{\gamma_{0}} \quad \text { with } \delta(0)=0 .
$$

Here $E_{\nu}(z)$ is the (complex) electric field amplitude at the frequency $\omega=\nu \omega_{0}$ near $\omega_{0}, \Delta \nu=\nu-1,|\Delta \nu| \ll 1$, and the Bessel function factor $[\mathrm{JJ}]=J_{0}(\xi)-J_{1}(\xi)$ with $\xi=$ $K_{0}^{2} /\left(4+2 K_{0}^{2}\right)$.

In the small signal regime before saturation, the electron distribution function can be decomposed into two parts: a coarse-averaged electron distribution function $V(\eta)$ (for a uniform bunch current) and a small perturbation containing the initial shot noise fluctuation and the FEL interaction $\delta F(\theta, \eta ; z)$. Incorporating the pendulum equations (4) and (5), the linearized Vlasov equation for the Fourier component of the distribution function $F_{\nu}(\eta ; z)=$ $\int \delta F(\theta, \eta ; z) \exp (-i \nu \theta) d \theta /(2 \pi)$ is

$$
\frac{d F_{\nu}}{d z}+i \nu 2 k_{u}(\eta+\delta) F_{\nu}+\frac{e K_{0}[\mathrm{JJ}]}{4 \gamma_{0}^{2} m c^{2}} E_{\nu}(z) e^{-i \Delta \nu k_{u} z} \frac{d V}{d \eta}=0 .
$$

The Maxwell equation for the electric field is then

$$
\frac{d E_{\nu}}{d z}=-\frac{e k_{0} K_{0}[\mathrm{JJ}]}{2 \epsilon_{0} \gamma_{0}} e^{i \Delta \nu k_{u} z} \int_{-\infty}^{\infty} d \eta F_{\nu}(\eta ; z),
$$

with $\epsilon_{0}$ being the vacuum permittivity.

The FEL equations (4), (5), and (8) are solved in perturbation theory when $\delta(z)$ can be considered small such as due to undulator errors [7]. Here we develop an approximate solution when $\delta(z)$ is not necessarily small but slowly varying with $z$.

\section{SOLUTION IN THE ONE-DIMENSIONAL CASE}

The Vlasov-Maxwell equations (7) and (8) can be solved by the Laplace transform when $\delta(z)=0[8,9]$. The system 
is characterized by the FEL Pierce parameter $\rho$ defined as [10]

$$
\rho=\left[\frac{1}{8 \pi} \frac{I_{e}}{I_{A}}\left(\frac{K_{0}[\mathrm{JJ}]}{1+K_{0}^{2} / 2}\right)^{2} \frac{\gamma_{0} \lambda_{0}^{2}}{\Sigma_{A}}\right]^{1 / 3},
$$

where $I_{e}$ is the electron peak current, $I_{A}=4 \pi \epsilon_{0} m c^{3} / e \approx$ $17 \mathrm{kA}$ is the Alfvén current, and $\Sigma_{A}$ is the area of the electron beam transverse cross section. For instance, the relative gain bandwidth $\Delta \nu$ is typically a few $\rho$, and the electric field gain length is about $\lambda_{u} /(4 \pi \rho)$. Since the main effect of the energy variation is to move electrons off resonance, $\delta(z)$ can be regarded as a slowly varying function of $z$ when

$$
\left|\frac{\lambda_{u}}{4 \pi \rho} \frac{d \delta}{d z}\right| \ll \text { a few } \rho \text { or }\left|\frac{\lambda_{u}}{4 \pi \rho} \frac{d \delta}{d z}\right|<\rho .
$$

This condition will allow us to use the WKB approximation well known in quantum mechanics (see, e.g., Ref. [11]) to solve Eqs. (7) and (8) and is satisfied if the accumulated energy change over the saturation distance (typically about 10 field gain length) is less than $10 \rho$.

\section{A. WKB approximation}

We first introduce the following dimensionless variables to simplify notation:

$$
\begin{aligned}
\bar{z} & =2 \rho k_{u} z, \quad \bar{\eta}=\frac{\eta}{\rho}=\frac{\gamma(z)-\gamma_{c}(z)}{\gamma_{0} \rho}, \\
\bar{\delta} & =\frac{\delta}{\rho}=\frac{\gamma_{c}(z)-\gamma_{r}(z)}{\gamma_{0} \rho}, \quad \bar{\nu}=\frac{\Delta \nu}{2 \rho}, \\
a_{\nu} & =-\frac{e K[\mathrm{JJ}]}{4 \gamma_{0}^{2} m c^{2} k_{u} \rho} e^{-i \Delta \nu k_{u} z} E_{\nu}, \quad f_{\nu}=\frac{2 k_{u} \rho^{2}}{k_{0}} F_{\nu} .
\end{aligned}
$$

Equations (8) and (7) in the matrix form are

$$
\frac{d}{d \bar{z}}\left(\begin{array}{c}
a_{\nu} \\
f_{\nu}
\end{array}\right)=i \mathrm{M}\left(\begin{array}{c}
a_{\nu}(\bar{z}) \\
f_{\nu}(\bar{\eta} ; \bar{z})
\end{array}\right)
$$

where

$$
\mathrm{M}=\left(\begin{array}{cc}
-\bar{\nu} & -i \int_{-\infty}^{\infty} d \bar{\eta} \\
-i \frac{d V}{d \bar{\eta}} & -[\bar{\eta}+\bar{\delta}(\bar{z})]
\end{array}\right)
$$

We define $\int_{-\infty}^{\infty} d \bar{\eta}$ as the integration operator that operates on a function of $\bar{\eta}$. Note that $\bar{\delta}(\bar{z})$ is slowly varying when $|d \bar{\delta} / d \bar{z}|<1$.

Following the general discussion of the WKB approximation using the matrix formalism presented in Appendix A, we seek a zeroth-order solution of Eq. (12) in the form

$$
\begin{aligned}
\exp \left(-i \int_{0}^{\bar{z}} \mu_{0}(\tau) d \tau\right) \Psi_{0} \equiv & \exp \left(-i \int_{0}^{\bar{z}} \mu_{0}(\tau) d \tau\right) \\
& \times\left(\begin{array}{c}
A_{0} \\
\mathcal{F}_{0}(\bar{\eta} ; \bar{z})
\end{array}\right) .
\end{aligned}
$$

In the $1 \mathrm{D}$ case, $A_{0}$ is simply a constant given by the initial conditions. Treating $d \mathcal{F}_{0} / d \bar{z}$ as a first-order term, the zeroth-order eigenvalue equation is

$$
\left(\begin{array}{cc}
\left(\mu_{0}-\bar{\nu}\right) & -i \int_{-\infty}^{\infty} d \bar{\eta} \\
-i \frac{d V}{d \bar{\eta}} & {\left[\mu_{0}-(\bar{\eta}+\bar{\delta}(\bar{z}))\right]}
\end{array}\right)\left(\begin{array}{c}
A_{0} \\
\mathcal{F}_{0}(\bar{\eta})
\end{array}\right)=0
$$

The eigenvalue is determined by solving the second row for

$$
\mathcal{F}_{0}(\bar{\eta} ; \bar{z})=\frac{i A_{0}}{\mu_{0}-[\bar{\eta}+\bar{\delta}(\bar{z})]} \frac{d V}{d \bar{\eta}}
$$

and inserting $\mathcal{F}_{0}$ into the first row. The dispersion relation for $\mu_{0}$ is

$$
\mu_{0}-\bar{\nu}=\int_{-\infty}^{\infty} \frac{d \bar{\eta}}{\left.\overline{[} \bar{\eta}+\bar{\delta}(\bar{z})-\mu_{0}\right]} \frac{d V}{d \bar{\eta}} .
$$

After changing variables to

$$
\hat{\mu}(\bar{z})=\mu_{0}(\bar{z})-\bar{\delta}(\bar{z}), \quad \hat{\nu}(\bar{z})=\bar{\nu}-\bar{\delta}(\bar{z}),
$$

we rewrite Eq. (17) as

$$
\hat{\mu}-\hat{\nu}=\int_{-\infty}^{\infty} \frac{d \bar{\eta}}{(\bar{\eta}-\hat{\mu})} \frac{d V}{d \bar{\eta}},
$$

which is the same FEL dispersion relation as in the constant-parameter case [8]. For a variable-parameter FEL, the instantaneous frequency detune $\hat{\nu}(\bar{z})=$ $\bar{\nu}-\bar{\delta}(\bar{z})$ is $\bar{z}$ dependent due to changes in the beam energy and the undulator parameter. As a result, the local growth rate $\operatorname{Im}\left(\mu_{0}\right)=\operatorname{Im}(\hat{\mu})$ is also a function of $\bar{z}$ (see Fig. 1). The corresponding eigenvector is

$$
\Psi_{0}(\bar{z})=\left(\begin{array}{c}
A_{0} \\
\mathcal{F}_{0}(\bar{\eta} ; \bar{z})
\end{array}\right) \propto\left(\begin{array}{c}
1 \\
\frac{i}{\mu_{0}-[\bar{\eta}+\bar{\delta}(\bar{z})]} \frac{d V}{d \bar{\eta}}
\end{array}\right) .
$$

To take into account the $z$ dependence of $\mathcal{F}_{0}$, we must include the first-order corrections for the eigenvalue and the eigenvector as

$$
\begin{aligned}
\left(\begin{array}{l}
a_{\nu} \\
f_{\nu}
\end{array}\right) \approx & \exp \left[-i \int_{0}^{\bar{z}}\left(\mu_{0}(\tau)+\mu_{1}(\tau)\right) d \tau\right]\left[\Psi_{0}(\bar{z})\right. \\
& \left.+\Psi_{1}(\bar{z})\right] .
\end{aligned}
$$

Note that both $\Psi_{1}=\left(A_{1}, \mathcal{F}_{1}(\bar{\eta})\right)$ and $\mu_{1}$ are considered small as compared to $\Psi_{0}$ and $\mu_{0}$, respectively, but the accumulated phase change $\int_{0}^{\bar{z}} \mu_{1}(\tau) d \tau$ in the exponent can be of the same order. Inserting Eq. (21) into Eq. (12), we obtain

$$
\begin{aligned}
& {\left[-i \mu_{0}(\bar{z})-i \mu_{1}(\bar{z})\right]\left(\Psi_{0}+\Psi_{1}\right)+\left(\Psi_{0}^{\prime}+\Psi_{1}^{\prime}\right)} \\
& \quad=i \mathrm{M}\left(\Psi_{0}+\Psi_{1}\right),
\end{aligned}
$$

where $\left({ }^{\prime}\right)=d / d \bar{z}$. Making use of $-i \mu_{0} \Psi_{0}=i \mathrm{M} \Psi_{0}$ and neglecting the higher-order terms $\mu_{1} \Psi_{1}$ and $\Psi_{1}^{\prime}$, we have 


$$
\begin{aligned}
& \Psi_{0}^{\prime}-i \mu_{1} \Psi_{0}=i\left(\mu_{0}+\mathrm{M}\right) \Psi_{1} \text { or } \\
& \left(\begin{array}{c}
0-i \mu_{1} \\
\frac{-i\left(\mu_{0}^{\prime}-\bar{\delta}^{\prime}\right)}{\left[\mu_{0}-(\bar{\eta}+\bar{\delta}(\bar{z}))\right]^{2}} \frac{d V}{d \bar{\eta}}+\frac{\mu_{1}}{\mu_{0}-[\bar{\eta}+\bar{\delta}(\bar{z})]} \frac{d V}{d \bar{\eta}}
\end{array}\right) \\
& =\left(\begin{array}{c}
i\left(\mu_{0}-\bar{\nu}\right) A_{1}+\int_{-\infty}^{\infty} d \bar{\eta} \mathcal{F}_{1}(\bar{\eta}) \\
A_{1} \frac{d V}{d \bar{\eta}}+i\left[\mu_{0}-(\bar{\eta}+\bar{\delta}(\bar{z}))\right] \mathcal{F}_{1}(\bar{\eta})
\end{array}\right) .
\end{aligned}
$$

The growth rate correction $\mu_{1}$ can be found by using an adjoint eigenvector and a properly defined scalar product as illustrated in Appendix B for the general 3D FEL system. In the $1 \mathrm{D}$ case, the adjoint eigenvector is simply

$$
\Phi_{0}=\left(1, \frac{i}{\mu_{0}-[\bar{\eta}+\bar{\delta}(\bar{z})]}\right)
$$

Defining the 1D scalar product as

$$
\begin{aligned}
\left\langle\Phi_{0} \mid \Psi_{0}\right\rangle_{1 \mathrm{D}} & =\left[1-\int_{-\infty}^{\infty} d \bar{\eta} \frac{d V / d \bar{\eta}}{\left[\mu_{0}-(\bar{\eta}+\bar{\delta}(\bar{z}))\right]^{2}}\right] \\
& \equiv B\left(\mu_{0}-\bar{\delta}\right),
\end{aligned}
$$

we apply $\Phi_{0}$ to both sides of Eq. (23). The resulting scalar product of the right-hand side with $\Phi_{0}$ is

$$
\begin{aligned}
& i\left(\mu_{0}-\bar{\nu}\right) A_{1}+\int_{-\infty}^{\infty} d \bar{\eta} \mathcal{F}_{1}(\bar{\eta}) \\
& +\int_{-\infty}^{\infty} d \bar{\eta}\left[\frac{i A_{1}}{\mu_{0}-[\bar{\eta}+\bar{\delta}(\bar{z})]} \frac{d V}{d \bar{\eta}}-\mathcal{F}_{1}(\bar{\eta})\right]=0
\end{aligned}
$$

in view of the dispersion relation Eq. (17). Thus, the scalar product of the left-hand side of Eq. (23) with $\Phi_{0}$ is

$$
\begin{aligned}
& -i \mu_{1} B\left(\mu_{0}-\bar{\delta}\right) \\
& \quad+\left(\mu_{0}^{\prime}-\bar{\delta}^{\prime}\right) \int_{-\infty}^{\infty} d \bar{\eta} \frac{d V / d \bar{\eta}}{\left[\mu_{0}-(\bar{\eta}+\bar{\delta}(\bar{z}))\right]^{3}}=0 .
\end{aligned}
$$

Using variables defined in Eq. (18), the correction to the complex growth rate is

$$
\begin{aligned}
\mu_{1} & =-i \frac{\mu_{0}^{\prime}-\bar{\delta}^{\prime}}{B\left(\mu_{0}-\bar{\delta}\right)} \int_{-\infty}^{\infty} d \bar{\eta} \frac{d V / d \bar{\eta}}{\left[\mu_{0}-(\bar{\eta}+\bar{\delta}(\bar{z}))\right]^{3}} \\
& =-i \frac{\hat{\mu}^{\prime}}{B(\hat{\mu})} \int_{-\infty}^{\infty} d \bar{\eta} \frac{d V / d \bar{\eta}}{(\hat{\mu}-\bar{\eta})^{3}},
\end{aligned}
$$

which can be obtained after solving the FEL dispersion relation [i.e., Eq. (17) or (19)].

For practical purposes, we assume a linear energy variation $\bar{\delta}(z)=\alpha \bar{z}$ with $|\alpha|<1$ for the WKB approximation to be valid. We consider both coherent amplification (for a seeded FEL) and self-amplified spontaneous emission.

\section{B. Coherent amplification}

Let us take a Gaussian energy distribution function

$$
V(\bar{\eta})=\frac{1}{\sqrt{2 \pi} \sigma_{\bar{\eta}}} \exp \left(\frac{-\bar{\eta}^{2}}{2 \sigma_{\bar{\eta}}^{2}}\right)
$$

with the rms energy spread $\sigma_{\bar{\eta}}$ in units of $\rho$, and define the plasma dispersion function

$$
D(\zeta)=\frac{1}{\sqrt{2 \pi}} \int_{C} d p \frac{p e^{-p^{2} / 2}}{p-\zeta},
$$

where the integration contour $C$ is from $p=-\infty$ to $\infty$ and is deformed so that the point $\zeta$ always stays above it in the complex $\zeta$ plane. Equation (19) becomes

$$
\hat{\mu}(\bar{z})-\hat{\nu}(\bar{z})+\frac{1}{\sigma^{2}} D\left(\frac{\hat{\mu}(\bar{z})}{\sigma}\right)=0,
$$

and Eq. (28) can be shown to be

$$
\mu_{1}=\left.i \frac{\alpha}{2 \sigma_{\bar{\eta}}^{4}} \frac{d^{2} D}{d \zeta^{2}}\left(1+\frac{1}{\sigma_{\bar{\eta}}^{3}} \frac{d D}{d \zeta}\right)^{-2}\right|_{\zeta=\hat{\mu}(\bar{z}) / \sigma_{\bar{\eta}}} .
$$

For a cold beam with a vanishing energy spread, we have $D(\zeta)=-\zeta^{-2}$, and

$$
\mu_{1}=-\frac{3 i \alpha}{\hat{\mu}^{4}\left(1+2 / \hat{\mu}^{3}\right)^{2}},
$$

where $\hat{\mu}-\hat{\nu}-\hat{\mu}^{-2}=0$ is the well-known cubic equation with a growing, a damping, and an oscillatory solution. At $\hat{\nu}=0$, we have $\hat{\mu}^{3}=1$ and

$$
\mu_{1}(\hat{\nu}=0)=-i \frac{\alpha}{3 \hat{\mu}} .
$$

In this case, the correction to the growing mode $\hat{\mu}^{(1)}=$ $\mu_{0}^{(1)}-\alpha \bar{z}=-1 / 2+i \sqrt{3} / 2$ is

$$
\mu_{1}^{(1)}=\frac{\alpha}{6}(-\sqrt{3}+i) .
$$

Therefore, the growth rate near $\hat{\nu}=\bar{\nu}-\alpha \bar{z}=0$ is increased (decreased) by $|\alpha| / 6$ for a linear energy gain (loss) with respect to the resonant energy. For a cold beam with constant beam and resonant energies, the maximum growth rate occurs when the electrons are on resonance and when a single growing mode dominates. However, as the electron energy is moving away from the resonance, slightly above resonance is the preferred situation since the energy modulation is immediately accompanied by the net energy loss of the electrons to the radiation field. Such an asymmetry also exists for the gain curve in a low-gain FEL [12].

The local growth rate of the radiation power predicted from the WKB approximation is compared to 1D FEL simulations with a seed signal. For a cold beam with a seed power $\left|a_{0}(0)\right|^{2}=10^{-6}$ initially on resonance (i.e., $\bar{\nu}=0$ ), the radiation power is completely specified by 
the growing, the damping, and the oscillatory modes and their corresponding corrections due to the energy variation, i.e.,

$$
\begin{aligned}
\bar{P}(\alpha ; \bar{z})= & \frac{\left|a_{0}(0)\right|^{2}}{9} \mid \sum_{n=1}^{3} \exp \left[-i \int_{0}^{\bar{z}} d \tau\left(\mu_{0}^{(n)}(\tau)\right.\right. \\
& \left.\left.+\mu_{1}^{(n)}(\tau)\right)\right]\left.\right|^{2} \cdot
\end{aligned}
$$

Here each mode has an initial amplitude $a_{0}(0) / 3$. Let us define the local power growth rate

$$
G(\alpha ; \bar{z}) \equiv \frac{d \ln [\bar{P}(\alpha ; \bar{z})]}{d \bar{z}} .
$$

Figure 2 shows the difference in the local growth rate $G(\alpha ; \bar{z})-G(0 ; \bar{z})$ for $\alpha= \pm 0.2$. The agreement between theory and simulation is very good. The initial growth rate is enhanced for a beam gaining energy relative to the resonant energy and is reduced for a beam losing energy. Nevertheless, at a larger undulator distance $(\bar{z}>2)$ when $\hat{\nu}=-\alpha \bar{z}(\bar{\nu}=0$ here $)$ is sufficiently detuned away from the resonance, the growth rates for both energy gain and loss are smaller than the growth rate when the beam energy stays on resonance.

For a beam with a Gaussian energy spread, we may only obtain an asymptotic solution in the high-gain regime since there are infinite damping modes during the initial power buildup [13]. We can still compare the local growth rate of the radiation power [i.e., Eq. (37)] derived from the 1D simulation with the dominant growing mode $2 \operatorname{Im}\left[\mu_{0}(\bar{z})+\right.$ $\left.\mu_{1}(\bar{z})\right]$ from the WKB approximation in the high-gain regime. Taking $\sigma_{\bar{\eta}}=0.5$ and seeding the FEL with $\left|a_{\bar{\nu}}(0)\right|^{2}=10^{-6}$ at the initial frequency detune $\bar{\nu} \approx-0.4$ that yields $2 \operatorname{Im}\left(\mu_{0}\right) \approx 1.4$ for a constant-parameter FEL, we show in Fig. 3 that the theory and the simulation agree fairly well for $\alpha= \pm 0.2$ in the high-gain regime when $\bar{z}>$ 5. In particular, the different growth rate between a beam

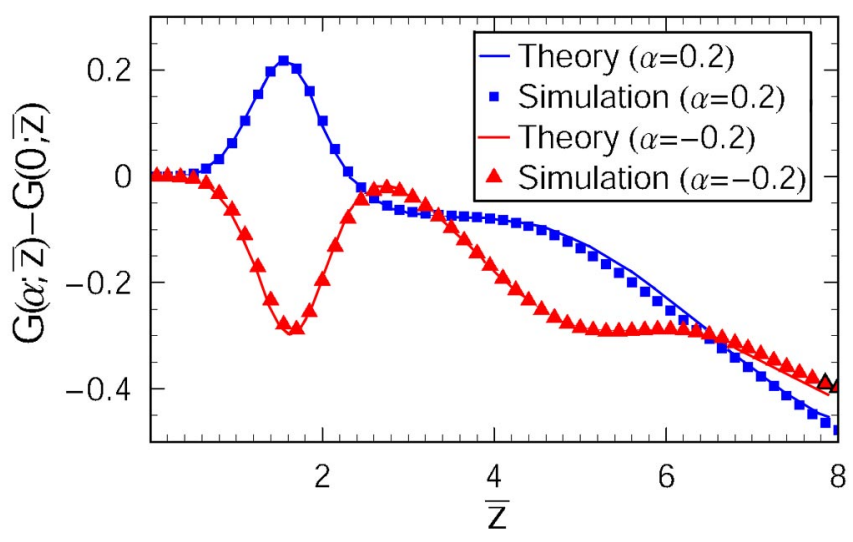

FIG. 2. (Color) Local power growth rate difference $G( \pm 0.2 ; \bar{z})-G(0 ; \bar{z})$ for a cold beam with an increasing (blue) or a decreasing (red) energy relative to the resonant energy in the undulator.

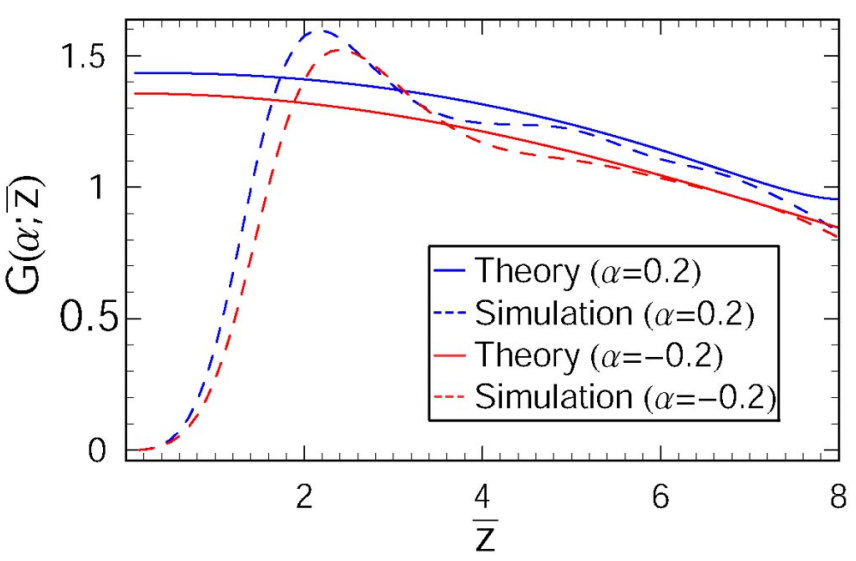

FIG. 3. (Color) Local power growth rate $G( \pm 0.2 ; \bar{z})$ for a beam with a Gaussian rms energy spread $\sigma_{\eta}=0.5 \rho\left(\sigma_{\bar{\eta}}=0.5\right)$ and with an increasing (blue) or a decreasing (red) centroid energy relative to the resonant energy in the undulator.

gaining and losing energy relative to the resonant energy is again due to the asymmetry discussed above.

\section{Self-amplified spontaneous emission}

The power spectrum for a constant-parameter SASE FEL in the high-gain regime has been determined in Refs. [8,9]. In the variable-parameter case discussed here, we can include the $\bar{z}$-dependent growth rate and its WKB correction for the growing mode as

$$
\frac{d P}{d \omega}=g_{S}(\bar{\nu}) \frac{\rho \gamma_{0} m c^{2}}{2 \pi} \exp \left[2 \int_{0}^{\bar{z}} d \tau \operatorname{Im}\left(\mu_{0}(\tau)+\mu_{1}(\tau)\right)\right]
$$

where

$$
g_{S}(\bar{\nu})=\frac{1}{\left|B\left(\mu_{0}(0)\right)\right|^{2}} \int_{-\infty}^{\infty} \frac{d \bar{\eta} V(\bar{\eta})}{\left|\mu_{0}(0)-\bar{\eta}\right|^{2}}
$$

$B\left(\mu_{0}\right)$ is defined in Eq. (25), and we have dropped the superscript (1) of the growing mode for simplicity. Equation (38) can be computed numerically for different frequencies to obtain the SASE spectrum as well as the total radiated power.

Because of the exponential growth, the radiation power in the high-gain regime is dominated when the frequency detune $\hat{\nu}=\bar{\nu}-\alpha \bar{z}$ is near the optimal value $\bar{\nu}_{m}$ that has the largest $\operatorname{Im}\left(\mu_{0}\right) \equiv \mu_{0 m}$. Let us expand

$$
\begin{aligned}
\operatorname{Im}\left(\mu_{0}\right) & \approx \mu_{0 m}\left[1-C_{2}\left(\hat{\nu}-\bar{\nu}_{m}\right)^{2}\right] \\
& =\mu_{0 m}\left[1-C_{2}\left(\bar{\nu}-\alpha \bar{z}-\bar{\nu}_{m}\right)^{2}\right],
\end{aligned}
$$

where $\mu_{0 m}=\sqrt{3} / 2, \bar{\nu}_{m}=0$, and $C_{2}=1 / 9$ for a cold beam and are shown in Figs. 4 and 5 for a Gaussian energy distribution. This expansion is expected to be valid if the accumulated change in $\hat{\nu}$ is less than the width of the frequency detune for the growth rate $\operatorname{Im}\left(\mu_{0}\right)$, i.e., when $|\alpha \bar{z}|<\sqrt{2 / C_{2}} \approx 4$. For a linear energy variation relative to 


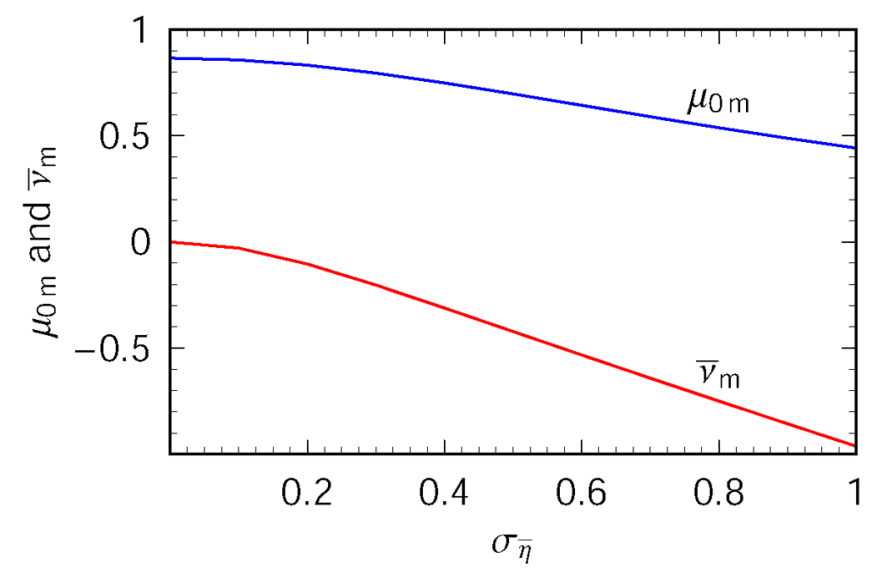

FIG. 4. (Color) Maximum zeroth-order growth rate $\mu_{0 m}$ (blue) and the optimal detune $\bar{\nu}_{m}$ (red) as a function of the rms energy spread $\sigma_{\bar{\eta}}$ (in units of $\rho$ ).

the resonant energy, the gain correction near $\hat{\nu}=\bar{\nu}_{m}$ can be factorized as

$$
\operatorname{Im}\left(\mu_{1}\right) \approx C_{\alpha} \mu_{0 m} \alpha
$$

Here $C_{\alpha}=\sqrt{3} / 9$ for a cold beam and is shown in Fig. 5 for a Gaussian energy distribution. Inserting Eqs. (40) and (41) into Eq. (38) and integrating over $\bar{z}$, we obtain

$$
\begin{aligned}
\frac{d P}{d \omega} \approx & g_{S}(\bar{\nu}) \frac{\rho \gamma_{0} m c^{2}}{2 \pi} \exp \left[2 \mu _ { 0 m } \int _ { 0 } ^ { \overline { z } } d \tau \left(1+C_{\alpha} \alpha\right.\right. \\
& \left.\left.-C_{2}\left(\bar{\nu}-\alpha \tau-\bar{\nu}_{m}\right)^{2}\right)\right] \\
= & \frac{\rho \gamma_{0} m c^{2}}{2 \pi} \exp \left[2 \mu_{0 m} \bar{z}\left(1+C_{\alpha} \alpha-C_{2} \frac{\alpha^{2} \bar{z}^{2}}{12}\right)\right] \\
& \times g_{S}(\bar{\nu}) \exp \left[-2 \mu_{0 m} C_{2} \bar{z}\left(\bar{\nu}-\bar{\nu}_{m}-\frac{\alpha \bar{z}}{2}\right)^{2}\right] .
\end{aligned}
$$

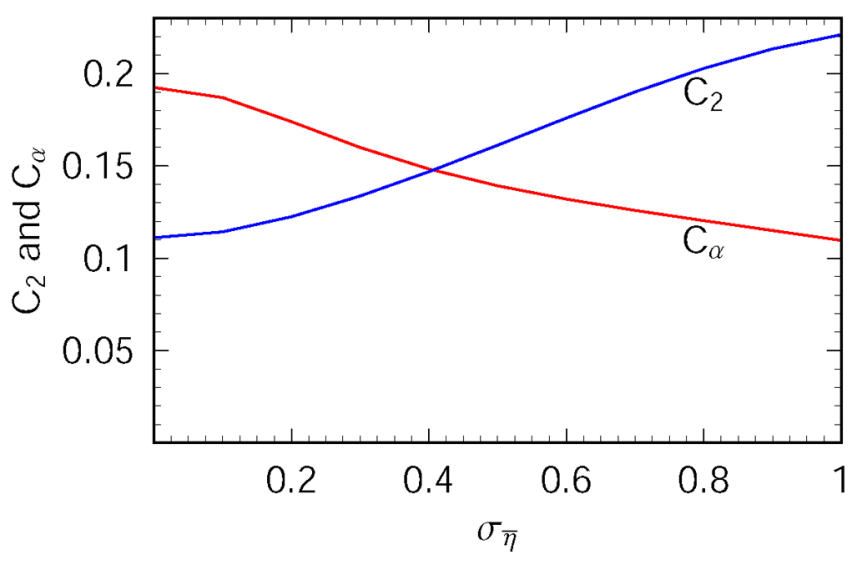

FIG. 5. (Color) Gain coefficients $C_{2}$ (blue) and $C_{\alpha}$ (red) defined in Eqs. (40) and (41) as a function of the rms energy spread $\sigma_{\bar{\eta}}$ (in units of $\rho$ ).
In general, $g_{S}(\bar{\nu})$ has a relatively weak dependence on the frequency detune $\bar{\nu}$ and is taken to be approximately constant from now on. Thus, the last exponent in Eq. (42) describes a Gaussian power spectrum that has basically the same rms bandwidth as a constant-parameter SASE, given by

$$
\begin{aligned}
\sigma_{\nu} & =\sigma_{\Delta \omega / \omega_{0}}=2 \rho \sigma_{\bar{\nu}}=\frac{\rho}{\sqrt{\mu_{0 m} C_{2} \bar{z}}} \\
& =\sqrt{\frac{\rho}{2 \mu_{0 m} C_{2} k_{u} z}}=\rho \sqrt{\frac{2 L_{G}}{C_{2} z}},
\end{aligned}
$$

with the power gain length $L_{G}=\lambda_{u} /\left(8 \pi \rho \mu_{0 m}\right)$. The central frequency of the power spectrum is determined by

$$
\bar{\nu}_{c}=\bar{\nu}_{m}+\alpha \bar{z} / 2=\bar{\nu}_{m}+\frac{\delta}{2 \rho} ;
$$

i.e., the central frequency of the radiation spectrum moves half as fast as does the optimal frequency (for the maximum zeroth-order growth rate) due to the changing energy. Finally, we integrate Eq. (42) over $\omega$ to obtain the total radiated power as

$P(z) \approx g_{S} \frac{\rho \gamma_{0} m c^{2}}{\sqrt{2 \pi}} \omega_{0} \sigma_{\nu} \exp \left[\frac{z}{L_{G}}\left(1+C_{\alpha} \alpha-C_{2} \frac{\alpha^{2} \bar{z}^{2}}{12}\right)\right]$

$$
=P_{m}(z) \exp \left[-\frac{1}{2}\left(\frac{\delta(z)-\delta_{m}(z)}{\sqrt{3} \sigma_{\nu}(z)}\right)^{2}\right],
$$

where $\delta(z)=\alpha \bar{z} \rho$ is the fractional energy change defined in Eq. (6),

$$
P_{m}(z)=g_{S} \frac{\rho \gamma_{0} m c^{2}}{\sqrt{2 \pi}} \omega_{0} \sigma_{\nu} \exp \left[\frac{z}{L_{G}}\left(1+\frac{3 C_{\alpha}^{2}}{4 \rho^{2} k_{u}^{2} z^{2} C_{2}}\right)\right]
$$

is the maximum SASE power under the optimal energy gain $\delta_{m}(z)=6 C_{\alpha} /\left(2 k_{u} z C_{2}\right)$, and the rms width of the fractional energy change for the SASE power is $\sqrt{3}$ times as large as the relative rms radiation bandwidth $\sigma_{\nu}$ determined by Eq. (43).

Equations (45) and (46) are valid for a slowly varying beam energy relative to the resonant energy in the highgain regime before saturation. In addition to the normal exponential growth given by the first term in the exponent of Eq. (45), the second term in the exponent describes the WKB correction to the growth rate and shows the gain enhancement when the beam increases energy relative to the resonant energy. The last term in the exponent of Eq. (45) shows the detuning effect of the energy variation that degrades the radiation power. The competition between a positive second term and a negative third term in the exponent of Eq. (45) leads to an optimal energy gain $\delta_{m}(z)$ in Eq. (46) that maximizes the output power. 


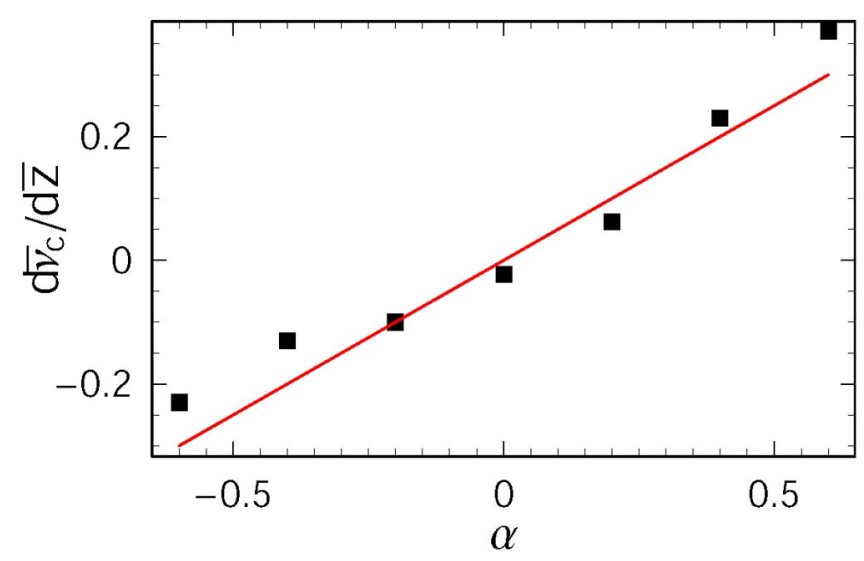

FIG. 6. (Color) Rate of the central frequency shift $d \bar{\nu}_{c} / d \bar{z}$ as a function of the energy gradient $\alpha$ in theory (solid line) and in simulations (symbols).

The linear theory is compared with the 1D SASE simulation results for a cold beam without any initial energy spread. Figure 6 shows that the rate of the central frequency shift extracted from the radiation phase in the simulation agrees well with the theoretical expectation $d \bar{\nu}_{c} / d \bar{z}=\alpha / 2$. The small discrepancy at larger $\alpha$ may come from the quadratic approximation used in Eq. (40). The scaled radiation power $\bar{P}=P /\left(\rho P_{\text {beam }}\right)$ for different energy gradient is computed with Eq. (46) using the simulated shot noise. Here $P_{\text {beam }}=I_{e} \gamma_{0} m c^{2} / e$ is the electron beam power. Figure 7 shows close agreement between theory and simulations for the dependence of the radiated power on the fractional energy variation at $\bar{z}=2 k_{u} \rho z=8$ before saturation.

Near the FEL saturation, the electron beam starts to lose a significant fraction of energy $(\sim \rho)$ to the radiation through the FEL interaction. In the case when the electrons gain energy relative to the resonant energy (i.e., for a small

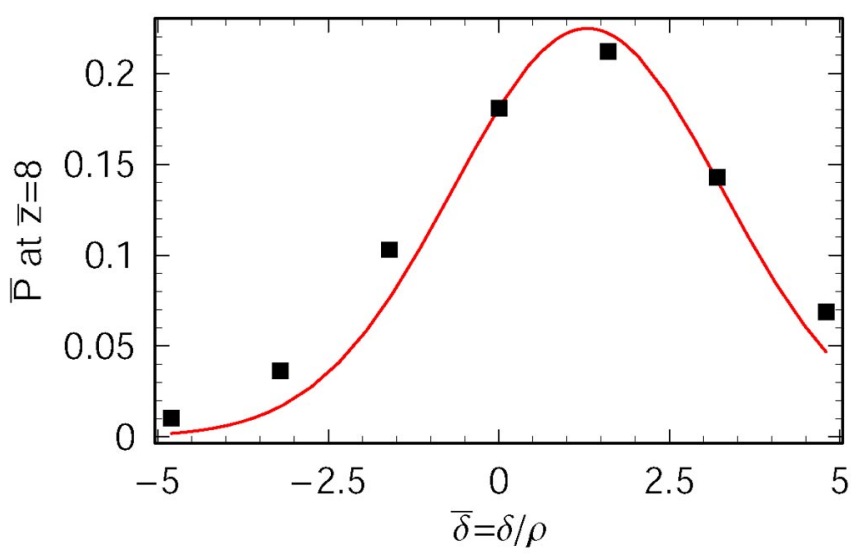

FIG. 7. (Color) SASE power (in units of $\rho P_{\text {beam }}$ ) at $\bar{z}=$ $2 \rho k_{u} z=8$ versus the fractional energy change $\bar{\delta}=\alpha \bar{z}=\delta / \rho$ from theory (curve) and from simulations (symbols) for a $1 \mathrm{D}$ cold beam.

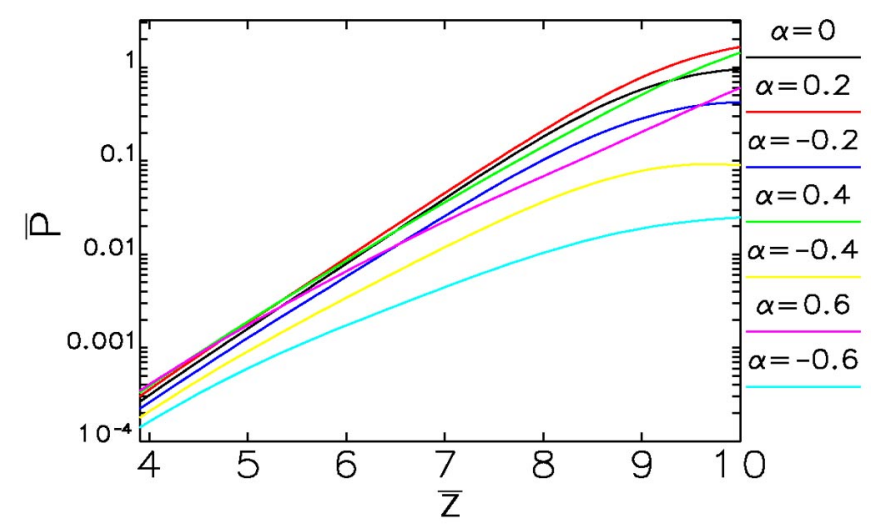

FIG. 8. (Color) Radiated power $\bar{P}$ (in units of $\rho P_{\text {beam }}$ ) as a function of the scaled undulator distance $\bar{z}=2 \rho k_{u} z$ for a cold beam in 1D SASE FEL simulations for different energy gradient $\alpha$.

and positive $\alpha$ ), the external energy gain compensates the FEL-induced energy loss and leads to longer resonant interaction between the electron beam and the radiation than in cases when $\alpha \leq 0$. 1D, cold beam SASE simulations in Fig. 8 show that $\alpha \approx 0.2$ (a fractional energy gain of about $2 \rho$ over the saturation distance at $\bar{z} \approx 10$ ) not only reduces the gain length in the linear regime, but also enhances the energy extraction efficiency in the nonlinear regime by about a factor of 2 .

\section{GENERALIZATION TO THE 3D SYSTEM}

The WKB solution to the coupled 3D Maxwell-Vlasov equations is given in Appendix B. In the high-gain regime where a single transverse mode with the largest growth rate $\operatorname{Im}\left(\mu_{0}\right)$ dominates over other higher-order modes, the SASE spectral power can be written as

$$
\begin{aligned}
\frac{d P}{d \omega}= & g_{S}(\bar{\nu}) \frac{\rho \gamma_{0} m c^{2}}{2 \pi}\left(\int_{-\infty}^{\infty} d \overline{\mathbf{x}}\left|A_{0}(\overline{\mathbf{x}}, \hat{\nu}(\bar{z}))\right|^{2}\right) \\
& \times \exp \left[2 \int_{0}^{\bar{z}} d \tau \operatorname{Im}\left(\mu_{0}(\tau)+\mu_{1}(\tau)\right)\right],
\end{aligned}
$$

where $g_{S}(\bar{\nu})$ is the expansion coefficient of the guided fundamental mode determined by the initial shot noise, $A_{0}(\overline{\mathbf{x}}, \hat{\nu})$ and $\mu_{0}=\hat{\mu}+\alpha \bar{z}$ are the dominant eigenmode and the eigenvalue at the instantaneous frequency detune $\hat{\nu}(z)=\bar{\nu}-\alpha \bar{z}$ determined by Eq. (B5), and the growth rate correction $\mu_{1}$ is given by (B11).

Following the approach developed in the 1D case, we consider the properties of $\mu_{0}$ and $\mu_{1}$ near the optimal detune and ignore the weak frequency dependencies of both $g_{S}$ and the transverse mode size. Thus, Eq. (46) for the SASE power is also valid in $3 \mathrm{D}$, i.e.,

$$
P(z) \approx P_{m}(z) \exp \left[-\frac{1}{2}\left(\frac{\delta(z)-\delta_{m}(z)}{\sqrt{3} \sigma_{\nu}(z)}\right)^{2}\right] .
$$

Here $\sigma_{\nu}(z)$ is the relative rms bandwidth of the guided 


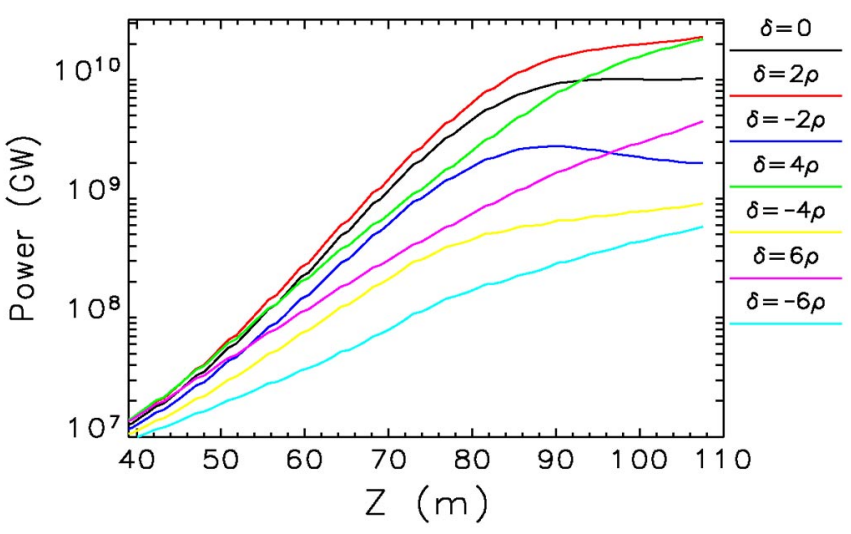

FIG. 9. (Color) LCLS power evolution obtained from GENESIS simulations for different fractional energy change $\delta(z=90 \mathrm{~m})$.

mode for a constant-parameter SASE found in the 3D theory or simulations. The optimal fractional energy gain $\delta_{m}(z)$ can be determined by the growth rate correction $\mu_{1}$ in the linear regime before saturation. For the maximum power at the saturation distance $z_{\text {sat }}$, 3D SASE simulation codes such as GINGER [14] and GENESIS [15] can be used to scan for $\delta_{m}\left(z_{\mathrm{sat}}\right)$. For example, using the standard LCLS parameters [2], GENESIS simulations shown in Fig. 9 indicate that $\delta_{m} \approx 2 \rho$ enhances the saturation power by about a factor of 2 at $z_{\text {sat }}=90 \mathrm{~m}$, very similar to the $1 \mathrm{D}$ results. Such a power enhancement has also been observed in start-to-end LCLS simulations including wakefield effects [16]. Since the expected rms bandwidth $\sigma_{\nu}$ near the LCLS saturation is very close to $\rho \approx 5 \times 10^{-4}$, we can estimate the FWHM fractional energy variation for the SASE power at saturation as

$$
(2 \sqrt{2 \ln 2}) \sqrt{3} \sigma_{\nu}\left(z_{\mathrm{sat}}\right) \approx 4 \sigma_{\nu}\left(z_{\mathrm{sat}}\right) \approx 4 \rho .
$$

Figure 10 shows the simulated LCLS power versus frac-

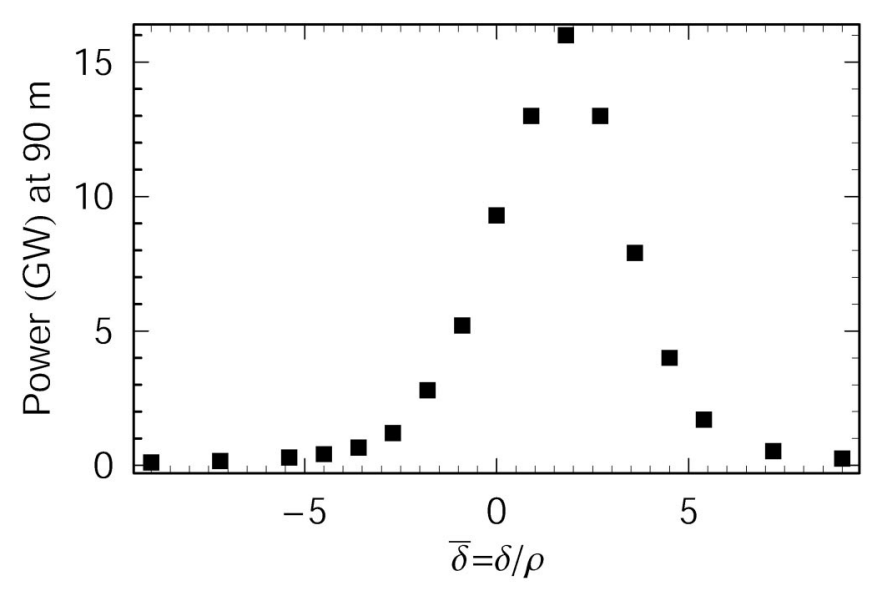

FIG. 10. LCLS power obtained from GENESIS simulations versus fractional energy change $\bar{\delta}=\delta / \rho$ at $z=90 \mathrm{~m}$. The maximum power is reached when $\delta \approx 2 \rho$, and the FWHM fractional energy change is about $4 \rho$, in agreement with Eq. (50). tional energy change $\delta$ at $z_{\text {sat }}=90 \mathrm{~m}$ with a FWHM nearly $4 \rho$.

\section{EFFECTS OF UNDULATOR WAKEFIELDS}

In this section we apply the above results to evaluate the SASE performance under the influence of undulator wakefields for the LCLS FEL at the resonant wavelength $\lambda_{0}=$ $1.5 \AA$ A Reference [1] discusses the ac resistive wall wakefield for both copper $(\mathrm{Cu})$ and aluminum $(\mathrm{Al})$ vacuum chambers with different geometries, generated by the expected LCLS bunch profile with two high-current horns at both the bunch head and tail for 1-nC bunch charge [see Fig. 5(c) of Ref. [1] for the current distribution]. The wakefield in the core part of the bunch (from the bunch coordinate $s \approx-30 \mu \mathrm{m}$ to $s \approx 0 \mu \mathrm{m}$ ) is of the most concern since this part of the bunch has the best beam quality in terms of emittance and energy spread and is responsible for most lasing. From Fig. 5(a) of Ref. [1], we see that the wakefield in this part of the bunch for $\mathrm{Cu}$ and $\mathrm{Al}$ with a standard round 5-mm diameter beam pipe may be approximated by

$$
\delta_{w}\left(z_{\mathrm{sat}}, s\right) \approx \delta_{A} \sin \left(\frac{2 \pi s}{\lambda_{\text {wake }}}\right)
$$

where $\delta_{A}$ is the fractional energy oscillation amplitude and is about $6 \rho(3 \rho)$ for $\mathrm{Cu}(\mathrm{Al})$ at the saturation distance $z_{\text {sat }} \approx 90 \mathrm{~m}$, and $\lambda_{\text {wake }} \approx 30 \mu \mathrm{m}$ is the wake oscillation period and is about half of the LCLS bunch length. In addition to compensating for the energy loss of the spontaneous radiation, we assume that the undulator parameter is tapered to produce a resonant energy change of $\delta_{r}=-2 \rho$ over $\sim 90-\mathrm{m}$ undulator distance, then the SASE power in the absence of any wakefield is optimized to yield $P_{m}\left(z_{\mathrm{sat}}\right) \approx 16 \mathrm{GW}$ from Fig. 10 instead of the nominal $8 \mathrm{GW}$ without any taper. Since such a wakefield creates negligible energy slopes (local energy chirps) over one cooperation length $\lambda_{0} /(4 \pi \rho)$, given by

$$
\begin{aligned}
\left|\frac{d \delta_{w}}{d s} \frac{\lambda_{0}}{4 \pi \rho}\right| & \leq \frac{2 \pi \delta_{A}}{\lambda_{\text {wake }}} \frac{\lambda_{0}}{4 \pi \rho} \leq \frac{12 \pi \rho}{30} \frac{1.5 \times 10^{-4}}{4 \pi \rho} \\
& =1.5 \times 10^{-5} \ll \rho,
\end{aligned}
$$

we ignore any local energy chirp and consider only the $z$-dependent energy variation for each FEL slice. The radiation power averaged over one wake oscillation period $\lambda_{\text {wake }} \approx 30 \mu \mathrm{m}$ in the core part of the bunch can be obtained by convoluting $\delta_{w}\left(z_{\mathrm{sat}}, s\right)-\delta_{r}=\delta_{w}\left(z_{\mathrm{sat}}, s\right)+$ $2 \rho$ with the SASE power response function Eq. (49), i.e.,

$$
\begin{aligned}
\frac{\left\langle P\left(z_{\text {sat }}\right)\right\rangle}{P_{m}\left(z_{\text {sat }}\right)} & =\int_{0}^{\lambda_{\text {wake }}} \frac{d s}{\lambda_{\text {wake }}} \exp \left[-\frac{1}{6} \frac{\delta_{w}\left(z_{\text {sat }}, s\right)^{2}}{\sigma_{\nu}^{2}\left(z_{\text {sat }}\right)}\right] \\
& =\exp \left[\frac{-\delta_{A}^{2}}{12 \sigma_{\nu}^{2}\left(z_{\text {sat }}\right)}\right] I_{0}\left[\frac{\delta_{A}^{2}}{12 \sigma_{\nu}^{2}\left(z_{\text {sat }}\right)}\right],
\end{aligned}
$$

where $I_{0}$ is the zeroth-order modified Bessel function. If we 


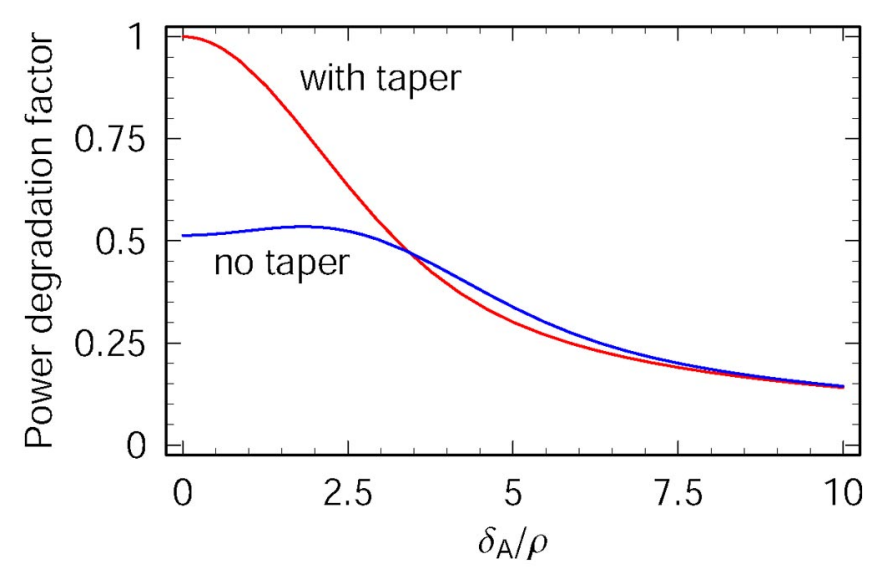

FIG. 11. (Color) Power degradation factor averaged over the core part of the bunch (with about $30 \mu \mathrm{m}$ in length) versus the sinusoidal wake oscillation amplitude $\delta_{A} / \rho$ at the LCLS saturation $(z=90 \mathrm{~m})$ for a prescribed tapered undulator (red) and without any taper (blue).

take $\sigma_{\nu}\left(z_{\mathrm{sat}}\right)=\rho$, then the averaged power degradation factor given by Eq. (53) is plotted in Fig. 11 (in red). Therefore, the average power in the core part of the bunch is about $50 \%(25 \%)$ of the maximum SASE power $(\approx$ $16 \mathrm{GW}$ at $90 \mathrm{~m}$ ) due to the ac resistive wall wakefield of a round 5-mm-diameter $\mathrm{Cu}(\mathrm{Al})$ vacuum pipe. As a comparison, Fig. 11 also shows the average power degradation without any taper (in blue), indicating the power improvement due to the above undulator taper is negated for a sinusoidal wake energy loss when its amplitude $\delta_{A} \geq 3 \rho$.

\section{CONCLUSION}

In this paper we present a self-consistent theory of a free electron laser with slowly varying beam and undulator parameters. A matrix formalism is developed to obtain the WKB solution of the Maxwell-Vlasov equations by employing the adjoint eigenvector that is orthogonal to the eigenvector of the dominant growing mode. This approach may be useful for other slowly varying processes in beam dynamics.

This theory is then applied to study the performance of a SASE FEL under a linear energy variation along the undulator distance. The optimal energy gain (or the equivalent undulator taper) for the maximum radiation power is determined in the linear regime through the WKB solution as well as at the saturation point through SASE simulations. For typical FEL parameters, we find that a fractional energy gain of about $2 \rho$ over the saturation distance enhances the saturated power by roughly a factor of 2 . Power degradation away from this optimal energy gain is approximately Gaussian given by Eq. (49), which is utilized to evaluate the LCLS performance under the influence of the ac resistive wall wakefield. The results discussed in this paper may be used to facilitate the design of a fourthgeneration x-ray source based on a high-gain FEL system.

\section{ACKNOWLEDGMENTS}

We would like to thank K. Bane, P. Emma, W. Fawley, S. Milton, H.-D. Nuhn, and S. Reiche for useful discussions and comments. This work was supported by Department of Energy Contract No. DE-AC02-76SF00515.

\section{APPENDIX A: WKB APPROXIMATION USING THE MATRIX FORMALISM}

We illustrate the WKB approximation using the matrix formalism here. Consider a second-order differential equation for a function $\phi(x)$ :

$$
\frac{d^{2} \phi}{d x^{2}}+k^{2}(x) \phi(x)=0,
$$

where the parameter $k(x)$ is assumed to be slowly varying in $x$. Equation (A1) can be regarded as the onedimensional, time-independent Schrödinger equation for the wave function $\phi(x)$. Let us convert Eq. (A1) to a couple of first-order differential equations as

$$
\frac{d \phi}{d x}=\varphi, \quad \frac{d \varphi}{d x}=-k^{2}(x) \phi .
$$

In the matrix form, we have

$$
\frac{d}{d x}\left(\begin{array}{l}
\phi \\
\varphi
\end{array}\right)=\mathrm{L}\left(\begin{array}{l}
\phi \\
\varphi
\end{array}\right) \quad \text { and } \quad \mathrm{L}=\left(\begin{array}{cc}
0 & 1 \\
-k^{2}(x) & 0
\end{array}\right)
$$

Since $k(x)$ is slowly varying, we expect the solution to closely approximate the free-particle state, i.e.,

$$
\left(\begin{array}{c}
\phi \\
\varphi
\end{array}\right) \approx \Psi_{0}(x) e^{i S_{0}(x)} \quad \text { with } \frac{d S_{0}}{d x}= \pm k(x) .
$$

The eigenvector given by $\pm i k \Psi_{0}=\mathrm{L} \Psi_{0}$ is

$$
\Psi_{0}^{+}(x)=\left(\begin{array}{c}
1 \\
i k(x)
\end{array}\right), \quad \Psi_{0}^{-}(x)=\left(\begin{array}{c}
1 \\
-i k(x)
\end{array}\right) .
$$

We define $\Phi_{0}$ as the adjoint eigenvector that satisfies $\pm i k \Phi_{0}=\Phi_{0} \mathrm{~L}$ and find

$$
\Phi_{0}^{+}(x)=\left(1, \frac{1}{i k(x)}\right), \quad \Phi_{0}^{-}(x)=\left(1,-\frac{1}{i k(x)}\right) .
$$

The adjoint eigenvector $\Phi_{0}^{ \pm}$is orthogonal to the eigenvector $\Psi_{0}^{ \pm}$since the scalar product

$$
\Phi_{0}^{ \pm} \Psi_{0}^{\mp}=0 \quad \text { and } \quad \Phi_{0}^{ \pm} \Psi_{0}^{ \pm}=2 .
$$

To take into account the slow variation of $\Psi_{0}(x)$ in Eq. (A4), we introduce first-order corrections as

$$
\left(\begin{array}{c}
\phi \\
\varphi
\end{array}\right) \approx\left[\Psi_{0}(x)+\Psi_{1}(x)\right] e^{i\left[S_{0}(x)+S_{1}(x)\right]},
$$

where $\Psi_{1}$ and $d S_{1} / d x$ are considered small, but not $S_{1}$. Inserting this into Eq. (A3) and ignoring higher-order terms $d \Psi_{1} / d x$ and $\Psi_{1} d S_{1} / d x$, we have 


$$
\frac{d \Psi_{0}}{d x}+i \frac{d S_{1}}{d x} \Psi_{0}=\left(-i \frac{d S_{0}}{d x}+\mathrm{L}\right) \Psi_{1} .
$$

Applying the adjoint eigenvector $\Phi_{0}$ to Eq. (A9), the scalar product of the right-hand side vanishes because $\Phi_{0}\left(i d S_{0} / d x\right)=\Phi_{0} L$, and the scalar product of the lefthand side becomes

$$
\Phi_{0}\left[\frac{d \Psi_{0}}{d x}+i \frac{d S_{1}}{d x} \Psi_{0}\right]=\frac{1}{k} \frac{d k}{d x}+2 i \frac{d S_{1}}{d x}=0,
$$

from which we obtain

$$
S_{1}=i \ln \sqrt{k(x)} .
$$

Inserting $S_{0}$ and $S_{1}$ into Eq. (A8) and neglecting $\Psi_{1}$ in comparison with $\Psi_{0}$, we obtain the WKB solution [11]

$$
\begin{aligned}
\phi(x) \approx & C_{1} \exp \left[i \int k(x) d x-\ln \sqrt{k(x)}\right] \\
& +C_{2} \exp \left[-i \int k(x) d x-\ln \sqrt{k(x)}\right] \\
= & \frac{C_{1}}{\sqrt{k(x)}} \exp \left[i \int k(x) d x\right]+\frac{C_{2}}{\sqrt{k(x)}} \\
& \times \exp \left[-i \int k(x) d x\right],
\end{aligned}
$$

where $C_{1}$ and $C_{2}$ are determined by initial and/or boundary conditions.

\section{APPENDIX B: GROWTH RATE FOR THE THREE- DIMENSIONAL FEL SYSTEM WITH SLOWLY VARYING BEAM AND UNDULATOR PARAMETERS}

We derive the (complex) growth rate for the threedimensional FEL system with slowly varying beam energy and undulator parameter using the WKB method discussed in Appendix A. Following the standard approach [17], the Maxwell-Vlasov equations including the radiation diffraction and the betatron motion are

$$
\begin{aligned}
& \frac{\partial}{\partial \bar{z}}\left(\begin{array}{c}
a_{\nu}(\overline{\mathbf{x}} ; \bar{z}) \\
f_{\nu}(\overline{\mathbf{x}}, \overline{\mathbf{p}}, \bar{\eta} ; \bar{z})
\end{array}\right)=i \mathrm{M}\left(\begin{array}{c}
a_{\nu} \\
f_{\nu}
\end{array}\right) \\
& \quad=\left(\begin{array}{c}
i\left(-\bar{\nu}+\frac{\bar{\nabla}_{\perp}^{2}}{2}\right) a_{\nu}+\int_{-\infty}^{\infty} d \overline{\mathbf{p}} \int_{-\infty}^{\infty} d \bar{\eta} f_{\nu} \\
a_{\nu} \frac{\partial f_{0}}{\partial \bar{\eta}}-i\left[\phi+\bar{\delta}(\bar{z})-i\left(\overline{\mathbf{x}}^{\prime} \frac{\partial}{\partial \overline{\mathbf{x}}}+\overline{\mathbf{p}}^{\prime} \frac{\partial}{\partial \overline{\mathbf{p}}}\right)\right] f_{\nu}
\end{array}\right),
\end{aligned}
$$

where $\mathbf{x}=(x, y)$ represents the transverse coordinates, $\overline{\mathbf{x}}=\mathbf{x} \sqrt{2 k_{0} k_{u} \rho}, \bar{\nabla}_{\perp}^{2}=\partial^{2} /\left(\partial \overline{\mathbf{x}}^{2}\right), \overline{\mathbf{x}}^{\prime}=d \overline{\mathbf{x}} / d \bar{z}=\overline{\mathbf{p}}, \overline{\mathbf{p}}^{\prime}=$ $d \overline{\mathbf{p}} / d \bar{z}=-\bar{k}_{\beta} \overline{\mathbf{x}}, \bar{k}_{\beta}=k_{\beta} /\left(2 k_{u} \rho\right)$ with $k_{\beta}=1 /\langle\beta\rangle$ being the average betatron wave number, $\phi=\bar{\eta}-\left(\overline{\mathbf{p}}^{2}+\right.$ $\left.\bar{k}_{\beta}^{2} \overline{\mathbf{x}}^{2}\right) / 2$, and $f_{0}\left(\overline{\mathbf{p}}^{2}+\bar{k}_{\beta}^{2} \overline{\mathbf{x}}^{2}, \bar{\eta}\right)$ is the average distribution function that is matched to the undulator focusing lattice. Note that $\bar{\delta}(\bar{z})=\left[\gamma_{c}(z)-\gamma_{r}(z)\right] /\left(\rho \gamma_{0}\right)$ describes the relative change of the beam energy to the resonant energy.
As illustrated in Sec. III and Appendix A, we seek a solution of Eq. (B1) in the form of Eq. (21), i.e.,

$$
\begin{aligned}
\Gamma & \equiv\left(\begin{array}{l}
a_{\nu} \\
f_{\nu}
\end{array}\right) \\
& \approx \exp \left[-i \int_{0}^{\bar{z}}\left(\mu_{0}(\tau)+\mu_{1}(\tau)\right) d \tau\right]\left[\Psi_{0}(\bar{z})+\Psi_{1}(\bar{z})\right] .
\end{aligned}
$$

The zeroth-order terms are given by

$$
\left(\mu_{0}+\mathrm{M}\right) \Psi_{0}(\bar{z})=0 \quad \text { with } \Psi_{0}=\left(\begin{array}{c}
A_{0}(\overline{\mathbf{x}} ; \bar{z}) \\
\mathcal{F}_{0}(\overline{\mathbf{x}}, \overline{\mathbf{p}}, \bar{\eta} ; \bar{z})
\end{array}\right) .
$$

From the second row of Eq. (B3), we obtain

$$
\mathcal{F}_{0}(\overline{\mathbf{x}}, \overline{\mathbf{p}}, \bar{\eta} ; \bar{z})=\frac{\partial f_{0}}{\partial \bar{\eta}} \int_{-\infty}^{0} d \tau A_{0}\left(\overline{\mathbf{x}}_{+} ; \bar{z}\right) e^{i\left(\phi+\bar{\delta}-\mu_{0}\right) \tau},
$$

where $\overline{\mathbf{x}}_{+}=\overline{\mathbf{x}} \cos \left(\bar{k}_{\beta} \tau\right)+\left(\overline{\mathbf{p}} / \bar{k}_{\beta}\right) \sin \left(\bar{k}_{\beta} \tau\right)$. If the energyshifted detune and growth rate defined in Eq. (18) are used, we obtain the same FEL eigenmode equation as in a constant-parameter FEL [18]:

$$
\begin{aligned}
(\hat{\mu} & \left.-\hat{\nu}+\frac{\bar{\nabla}_{\perp}^{2}}{2}\right) A_{0}(\overline{\mathbf{x}} ; \bar{z}) \\
& =i \int_{-\infty}^{\infty} d \overline{\mathbf{p}} \int_{-\infty}^{\infty} d \bar{\eta} \int_{-\infty}^{0} d \tau A_{0}\left(\overline{\mathbf{x}}_{+} ; \bar{z}\right) e^{i(\phi-\hat{\mu}) \tau} \frac{\partial f_{0}}{\partial \bar{\eta}} .
\end{aligned}
$$

Here $\hat{\nu}(\bar{z})=\bar{\nu}-\bar{\delta}(\bar{z})$ is a $\bar{z}$-dependent frequency detune because of the energy change. As a result, both $\hat{\mu}(\hat{\nu}(\bar{z}))=$ $\mu_{0}-\bar{\delta}(z)$ and $A_{0}=A_{0}(\overline{\mathbf{x}}, \hat{\nu}(\bar{z}))$ are functions of $\bar{z}$ determined by Eq. (B5).

To take into account the next-order correction in the complex growth rate, we make use of the adjoint eigenvector introduced in Refs. [19-21] for the initial value solution of the 3D FEL system because M in Eq. (B1) is a non-Hermitian operator [17]. Defining the scalar product of two arbitrary vectors $\Gamma_{1}$ and $\Gamma_{2}$ as $[19,20]$

$$
\begin{aligned}
\left\langle\Gamma_{1} \mid \Gamma_{2}\right\rangle= & \int_{-\infty}^{\infty} d \overline{\mathbf{x}} a_{\nu 1} a_{\nu 2} \\
& +\int_{-\infty}^{\infty} d \overline{\mathbf{x}} \int_{-\infty}^{\infty} d \overline{\mathbf{p}} \int_{-\infty}^{\infty} d \bar{\eta} f_{\nu 1} f_{\nu 2},
\end{aligned}
$$

we construct an adjoint eigenvector $\Phi_{0}=\left(\tilde{A}_{0}, \tilde{\mathcal{F}}_{0}\right)$ so that it is orthogonal to $\Psi_{0}$, i.e.,

$$
\left\langle\Phi_{0}^{(m)} \mid \Psi_{0}^{(n)}\right\rangle=\delta_{n m}\left\langle\Phi_{0}^{(n)} \mid \Psi_{0}^{(n)}\right\rangle .
$$

Here the index $n=1,2,3, \ldots$ indicates a discrete set of eigenvalues $\mu_{0}^{(n)}$ and eigenvectors $\Psi_{0}^{(n)}$ that satisfy Eq. (B5), and $\delta_{n m}$ is the Kronecker delta function. We also designate the first mode $(n=1)$ to be the growing mode with the largest growth rate $\operatorname{Im}\left(\mu_{0}\right)$. It can be shown 
that the eigenvalue corresponding to the adjoint eigenvector is also $\hat{\mu}$, and that $\tilde{A}_{0}=A_{0}[19-21]$ and

$$
\tilde{\mathcal{F}}_{0}=\int_{-\infty}^{0} d \tau A_{0}\left(\overline{\mathbf{x}}_{-}\right) e^{i\left(\phi+\bar{\delta}-\mu_{0}\right) \tau}
$$

with $\overline{\mathbf{x}}_{-}=\overline{\mathbf{x}} \cos \left(\bar{k}_{\beta} \tau\right)-\left(\overline{\mathbf{p}} / \bar{k}_{\beta}\right) \sin \left(\bar{k}_{\beta} \tau\right)$.

Assuming the set of eigenvectors is complete, we can expand the first-order correction $\Psi_{1}$ in Eq. (B2) as $\Psi_{1}=$ $\sum_{n} \kappa_{n} \Psi_{0}^{(n)}$. Inserting Eq. (B2) into Eq. (B1) and dropping the higher-order terms $\mu_{1} \Psi_{1}$ and $\Psi_{1}^{\prime}$, we obtain

$$
\begin{aligned}
\Psi_{0}^{\prime}-i \mu_{1} \Psi_{0} & =i\left(\mu_{0}+\mathrm{M}\right) \Psi_{1}=i\left(\mu_{0}+\mathrm{M}\right) \sum_{n} \kappa_{n} \Psi_{0}^{(n)} \\
& =i \sum_{n}\left(\mu_{0}-\mu_{0}^{(n)}\right) \kappa_{n} \Psi_{0}^{(n)}
\end{aligned}
$$

Since we are interested in the high-gain behavior when the first mode with its largest growth rate dominates, we take $\mu_{0}=\mu_{0}^{(1)}$ and apply its corresponding adjoint eigenvector to form the scalar product at both sides of Eq. (B9). The scalar product of the right-hand side vanishes in view of the orthogonality relation of Eq. (B7), and the scalar product of the left-hand side is

$$
\left\langle\Phi_{0} \mid \Psi_{0}^{\prime}-i \mu_{1} \Psi_{0}\right\rangle=0
$$

Thus, the first-order correction to the complex growth rate for the 3D FEL system is

$$
\mu_{1}=-i \frac{\left\langle\Phi_{0} \mid \Psi_{0}^{\prime}\right\rangle}{\left\langle\Phi_{0} \mid \Psi_{0}\right\rangle}
$$

It is straightforward to show that Eq. (B11) reduces to Eq. (28) in the 1D case since $A_{0}(\overline{\mathbf{x}})$ becomes independent of $\overline{\mathbf{x}}$ and the scalar product $\left\langle\Phi_{0} \mid \Psi_{0}\right\rangle=\Sigma_{A}\left\langle\Phi_{0} \mid \Psi_{0}\right\rangle_{1 \mathrm{D}}$, where $\Sigma_{A}$ is the area of the electron beam transverse cross section and $\left\langle\Phi_{0} \mid \Psi_{0}\right\rangle_{1 \mathrm{D}}$ is given in Eq. (25).

[1] K. Bane and G. Stupakov, SLAC Report No. SLAC-PUB10707, 2004.

[2] SLAC Linac Coherent Light Source (LCLS) Conceptual Design Report No. SLAC-R-593, 2002.

[3] N. Kroll, P. Morton, and M. Rosenbluth, IEEE J. Quantum Electron. 17, 1436 (1981).

[4] H.-D. Nuhn, Nucl. Instrum. Methods Phys. Res., Sect. A 429, 249 (1999).

[5] S. Reiche and H. Schlarb, Nucl. Instrum. Methods Phys. Res., Sect. A 445, 155 (2000).

[6] W. Colson, Phys. Lett. A 64, 190 (1977).

[7] L.-H. Yu, S. Krinsky, R. Gluckstern, and J. van Zeijts, Phys. Rev. A 45, 1163 (1992).

[8] K.-J. Kim, Nucl. Instrum. Methods Phys. Res., Sect. A 250, 396 (1986).

[9] J.-M. Wang and L.-H. Yu, Nucl. Instrum. Methods Phys. Res., Sect. A 250, 484 (1986).

[10] R. Bonifacio, C. Pellegrini, and L. Narducci, Opt. Commun. 50, 373 (1984).

[11] R. Liboff, Introductory Quantum Mechanics (AddisonWesley, Reading, MA, 1980).

[12] J. Madey, Nuovo Cimento Soc. Ital. Fis. 50B, 64 (1979).

[13] E. Saldin, E. Schneidmiller, and M. Yurkov, The Physics of Free Electron Lasers (Springer-Verlag, Berlin, 1999).

[14] W. Fawley, LBL Report No. LBNL-49625, 2002.

[15] S. Reiche, Nucl. Instrum. Methods Phys. Res., Sect. A 429, 243 (1999).

[16] W. Fawley and S. Reiche (private communication).

[17] K.-J. Kim, Phys. Rev. Lett. 57, 1871 (1986).

[18] L.-H. Yu, S. Krinsky, and R. Gluckstern, Phys. Rev. Lett. 64, 3011 (1990).

[19] K.-J. Kim (unpublished).

[20] Z. Huang and K.-J. Kim, Phys. Rev. E 62, 7295 (2000).

[21] M. Xie, Nucl. Instrum. Methods Phys. Res., Sect. A 475, 51 (2001). 